\title{
Estrutura Fatorial da Versão Brasileira do California Older Person's Pleasant Events Schedule ${ }^{1}$
}

\author{
Heloísa Gonçalves Ferreira² \\ Universidade Federal do Triângulo Mineiro, Uberaba-MG, Brasil \\ Elizabeth Joan Barham \\ Universidade Federal de São Carlos, São Carlos-SP, Brasil
}

RESUMO

O objetivo do presente estudo foi investigar evidências de validade da estrutura interna da versão brasileira do California Older Person Pleasant Events Schedule - OPPES-BR, um instrumento para avaliar o envolvimento de idosos em atividades prazerosas, que pode ser utilizado em contexto clínico para o tratamento da depressão. Para tal, foi realizada uma análise fatorial exploratória em amostra brasileira de 337 idosos não institucionalizados e sem alterações cognitivas, com idade média de 69,7 anos, sendo 68,2\% do sexo feminino. Observou-se que o OPPES-BR abarcou quatro domínios: Atividades Sociais e de Competência, Atividades Contemplativas, Atividades Práticas e Atividades Intelectuais, explicando 43,6\% da variância total. Essa estrutura fatorial necessita ainda ser confirmada em uma segunda amostra de idosos brasileiros, por meio de análises fatoriais confirmatórias. No entanto, os resultados obtidos já fornecem evidências de validade da estrutura interna do OPPES-BR, sugerindo possibilidades de aplicação em contextos de intervenções e de pesquisas com idosos brasileiros.

Palavras-chave: análise fatorial; validade de construto; escala; idosos.

\section{ABSTRACT - Factor Structure of the Brazilian Version of the California Older Person's Pleasant Events Schedule}

The objective of the present study was to investigate internal structure validity evidence of the Brazilian version of the California Older Person's Pleasant Events Schedule (COPPES-BR), an instrument to evaluate the involvement of older adults in pleasurable activities, which can be used in clinical context for the treatment of depression. To that end, an exploratory factor analysis was performed in a Brazilian sample of 337 non-institutionalized older adult individuals with no cognitive impairment, with a mean age of 69.7 years, of which $68.2 \%$ were female. It was observed that COPPES-BR covered four domains: Social Activities and Competence, Contemplative Activities, Practical Activities, and Intellectual Activities, accounting for $43.6 \%$ of the total variance. This factor structure still needs to be confirmed in a second sample of Brazilian seniors through confirmatory factor analysis. However, the results obtained already provide validity evidence of the internal structure of COPPES-BR, suggesting possibilities of application in contexts of interventions and research with Brazilian seniors.

Keywords: factor analysis; construct validity; scale; seniors; older adults; elderly.

\section{RESUMEN - Estructura Factorial de la Versión Brasileña de California Older Person's Pleasant Events Schedule}

El objetivo de este estudio fue investigar evidencias de validez en la estructura interna de la versión brasileña del California Older Person Pleasant Events Schedule - OPPES-BR, un instrumento norteamericano que evalúa la participación de los ancianos en actividades placenteras, que puede ser utilizado en el contexto clínico para el tratamiento de depresión. Para eso fue realizado un análisis factorial exploratorio con una muestra brasileña de 337 ancianos de la comunidad, sin alteraciones cognitivas, con edad promedio de 69,7 años, siendo 68,2\% de sexo femenino. Se observó que el OPPES-BR incluye cuatro dominios: Actividades Sociales y de Competencia, Actividades Contemplativas, Actividades Prácticas y Actividades Intelectuales, explicando un 43,6\% de la varianza total. Esta estructura factorial necesita aún de confirmación con una segunda muestra de ancianos brasileños, a través de análisis factorial confirmatorio. Sin embargo, los resultados obtenidos proporcionan evidencias de validez de estructura interna del OPPES-BR, sugiriéndose posibilidades de aplicación en contextos de intervención y de investigación con ancianos brasileños.

Palabras clave: análisis factorial; validez de constructo; instrumento; ancianos.

Pesquisadores têm apontado necessidades de se planejar ações direcionadas ao tratamento e prevenção da depressão na população idosa brasileira, uma vez que existe uma alta prevalência do transtorno, que variou entre $30,6 \%$ a $34,4 \%$ entre idosos brasileiros da comunidade (Alvarenga, Oliveira, \& Faccenda, 2012; Nogueira, 
Rubin, Giacobbo, Gomes, \& Neto, 2014). Observa-se, ainda, uma tendência crescente na proporção de idosos deprimidos à medida que eles se tornam mais dependentes fisicamente (Lima, Silva, \& Ramos, 2009).

A relação entre depressão em idosos e baixo envolvimento em atividades prazerosas tem sido bastante documentada na literatura desde a década de 70 , quando iniciaram-se os primeiros estudos para identificar técnicas psicoterapêuticas para tratar depressão em idosos no contexto da psicoterapia comportamental e cognitivo-comportamental (Lewinsohn \& Graf, 1973; Bouman \& Luteijn, 1986; Rider, Gallagher-Thompson, \& Thompson, 2004; Meeks, Young, \& Looney, 2007). O modelo comportamental da depressão assume que o transtorno é causado por uma baixa taxa de respostas capazes de gerar reforçadores positivos, ou seja, indivíduos depressivos não têm um envolvimento suficiente em atividades que geram sentimentos positivos, sendo que reforçadores positivos correspondem aos eventos que o indivíduo avalia como sendo prazerosos (Meeks et al., 2007).

$\mathrm{Na}$ década de 70 , pesquisadores interessados em tratar a depressão em idosos pautados nos conceitos teóricos da Análise do Comportamento criaram instrumentos para avaliar o envolvimento de idosos em atividades agradáveis. O construto acessado por essas escalas foi definido como sendo uma medida aproximada de eventos reforçadores (Lewinsohn \& Graf, 1973).

O California Older Person's Pleasant Events Schedule (Rider et al., 2004, 2016) foi uma das escalas construídas e adaptadas para avaliar envolvimento em atividades prazerosas por idosos, sendo um instrumento útil na tarefa de avaliar o envolvimento inicial do idoso em atividades prazerosas e identificar formas de ampliar esse envolvimento. Trata-se de um instrumento americano utilizado em contexto clínico no tratamento da depressão em idosos. Ele é composto por 66 itens que são avaliados usando duas subescalas de pontuação: (a) a subescala de Frequência é usada para indicar a frequência de prática de cada atividade; e (b) a subescala de Agradabilidade é usada para registrar o prazer experimentado na prática da atividade, ou o prazer que o idoso teria experimentado caso tivesse realizado a atividade.

O COPPES é um instrumento composto por itens adequados à população idosa que representam atividades que ela normalmente avalia como sendo prazerosas (Hedlund \& Gilewski, 1980, citado por Rider et al., 2004). Posteriormente, usando uma amostra de idosos americanos provenientes da Califórnia, Rider et al. (2004) definiram a estrutura fatorial do instrumento em domínios ou categorias de atividades que idosos normalmente apreciam estar envolvidos. A partir dessas análises, foi identificado que as atividades descritas nos itens do instrumento pertencem a cinco domínios: (a) socializar (socializing), que descreve atividades que objetivam interações sociais, tais como fazer amigos, expressar afeto e ser gentil com os outros; (b) relaxar (relaxing), que abarca atividades que captam interesses do indivíduo, de foco externo, como entrar em contato com a natureza, ler e explorar novas áreas; (c) contemplar (contemplating), que inclui atividades realizadas pelo indivíduo com foco interno, como meditar, pensar sobre si e sobre os outros; (d) ser efetivo (being effective), que descreve atividades que permitem que a pessoa derive um senso de competência individual; e (e) ser ativo (doing), que inclui atividades que os idosos geralmente encaram como hobbies e projetos. O índice de consistência interna dos domínios (alfa de Cronbach) variou de 0,63 a 0,86 , que são valores considerados como adequados a muito bons. O escore no instrumento ainda apresentou correlação negativa significativa com depressão, indicando evidências de validade com base nas relações com variáveis externas (Rider et al., 2004, 2016).

Aplicando-se o instrumento, é possível verificar em quais domínios o cliente apresenta maior ou menor grau de envolvimento e qual o nível de prazer associado com cada atividade. A partir dessas informações, traça-se um plano de intervenção individualizado que tem por objetivo criar condições necessárias para que o cliente aumente a frequência da prática de atividades classificadas como prazerosas, porém, que relata praticar com baixa frequência. O instrumento também é útil para ajudar o cliente a discriminar em quais domínios de atividades experimenta maior ou menor prazer, servindo de guia para que o idoso e terapeuta foquem seus esforços para aumentar a frequência de atividades nos domínios que forem mais relevantes para cada cliente.

No contexto brasileiro, Ferreira e Barham (2011) constataram a ausência de instrumentos psicométricos que avaliem o envolvimento de idosos em atividades prazerosas. Após um exame dos instrumentos existentes no cenário internacional, escolherem o COPPES, que já foi submetido a um processo de adaptação transcultural para uso no Brasil, onde o instrumento original foi traduzido por profissionais brasileiros da área com fluência em inglês, retrotraduzido por nativos da língua inglesa com fluência em português, e seus itens foram avaliados por um comitê de especialistas, apresentando ao final desse processo, equivalências semântica, idiomática e cultural satisfatórias (Ferreira \& Barham, 2013).

A versão resultante do instrumento após a adaptação transcultural foi nomeada como OPPES-BR (Older Person Pleasant Events Schedule - versão brasileira) e já foi submetida a uma Análise Fatorial Confirmatória (AFC) (Ferreira, Barham, \& Fontaine, 2015). Neste estudo, embora observou-se relações significativas entre o OPPES-BR com depressão (evidências de validade externa) e excelente consistência interna entre todos os itens do instrumento (evidências de validade interna), constatou-se também que a estrutura fatorial original não apresentou índices de ajustamento adequados à amostra brasileira para a subescala Frequência $\left(\chi^{2} / d f=2,57\right.$; CFI $=.728 ; \quad$ GFI $=0,627 ; \quad$ RMSEA $=0,07 ; \quad p[$ RMSEA $<$ 
$0,05]<0,001)$ e para a subescala Agradabilidade $\left(\chi^{2} / d f=2,167 ; \mathrm{CFI}=0,795 ; \mathrm{GFI}=0,682 ; \mathrm{RMSEA}=0,060\right.$; $p$ [RMSEA <.05]<.001), levando-se em conta os valores de referência propostos por Maroco (2010). Além disso, os domínios do instrumento apresentaram correlações muito altas entre si e os índices de modificação sugeriram correlações entre resíduos e correlações entre resíduos e fatores, indicando que uma porção considerável do comportamento dos itens não era explicada pela estrutura fatorial original. De acordo com Reichenheim, Hokerberg, e Moraes (2014), quando anomalias desses tipos são observadas, ao invés de fazer os ajustes sugeridos pelos índices de modificação, seria mais recomendado partir para uma abordagem exploratória de uma nova estrutura fatorial para o instrumento.

Logo, diante de tais constatações, torna-se necessária a realização de uma análise fatorial exploratória (AFE) para identificar quais são as categorias de atividades abarcadas pelo OPPES-BR que melhor se ajustam à amostra de idosos brasileiros e, dessa forma, investigar mais evidências de validade da versão brasileira do instrumento, com relação a sua estrutura interna. Diante da alta prevalência de depressão na população idosa brasileira e considerando a necessidade de diagnosticar e tratar adequadamente este transtorno em idosos (Lima et al., 2009; Nogueira et al., 2014), contar com instrumentos adaptados ao contexto brasileiro que possam ser usados em intervenções psicoterapêuticas para tratar a depressão em idosos seria extremamente importante. Considerando que o COPPES já foi adaptado para a cultura brasileira (Ferreira \& Barham, 2013) e que os primeiros estudos que investigaram evidências de validade da versão brasileira demonstraram resultados satisfatórios (Ferreira et al., 2015), torna-se relevante continuar com o processo de validação da escala para o Brasil.

\section{Objetivos}

Logo, o objetivo deste estudo foi verificar se o OPPES-BR apresenta evidências de validade de sua estrutura interna, especificamente por meio da realização de análises fatoriais exploratórias, para, então, investigar os domínios abarcados pelo instrumento quando aplicado em idosos brasileiros.

\section{Método}

\section{Aspectos Éticos}

Este projeto de pesquisa foi encaminhado para o Comitê de Ética em Pesquisa da Universidade Federal de São Carlos, tendo sido aprovado (CAAE: 00713712.4.0000.5504).

\section{Participantes}

Participaram deste estudo 337 idosos não institucionalizados, residentes em São Carlos, uma cidade de médio porte do estado de São Paulo. Os idosos foram selecionados de acordo com a distribuição do Índice Paulista de Vulnerabilidade Social (IPVS) em São Carlos, com o objetivo de garantir a variabilidade socioeconômica da amostra. Além disso, buscou-se atingir uma proporção de distribuição do IPVS na amostra, que fosse semelhante à distribuição do IPVS em São Carlos.

A vulnerabilidade social é um construto multidimensional que diz respeito ao processo de estar em risco para redução da qualidade de vida, em função do contexto de moradia, resultante de condições potencialmente inadequadas nos planos econômico, social, psicológico, familiar, cognitivo ou físico (Fundação Seade, 2008). Segundo o IPVS, foram estabelecidos seis níveis de vulnerabilidade social: 1 - nenhuma vulnerabilidade; 2 - vulnerabilidade muito baixa; 3 - vulnerabilidade baixa; 4 - vulnerabilidade média; 5 - vulnerabilidade alta; 6 - vulnerabilidade muito alta.

Na Tabela 1, pode ser visualizada a distribuição percentual dos respondentes do presente estudo e da composição da população no município de São Carlos, segundo o IPVS.

Tabela 1

Distribuição dos Participantes deste Estudo e da População no Município de São Carlos, segundo o Índice Paulista de Vulnerabilidade Social (IPVS) de sua Residência

\begin{tabular}{ccc}
\hline IPVS & $\begin{array}{c}\text { Amostra do estudo } \\
(\%)\end{array}$ & $\begin{array}{c}\text { População de } \\
\text { São Carlos (\%) }\end{array}$ \\
\hline 1 & 12,5 & 8,7 \\
2 & 39,9 & 36,3 \\
3 & 23,4 & 24,8 \\
4 & 14,0 & 17,5 \\
5 & 6,5 & 8,4 \\
6 & 3,7 & 4,2 \\
Total & 100 & 100 \\
\hline
\end{tabular}

A idade média dos participantes foi de 69,7 anos, com idades variando de 60 a 93 anos $(D P=7,31)$. Em relação ao sexo, $68,2 \%$ da amostra era do sexo feminino, e $31,8 \%$ era do sexo masculino. Em relação à escolaridade, $11,5 \%$ dos idosos não sabia ler ou escrever e 44,8\% da amostra apresentava ensino fundamental incompleto. Com relação à renda, $77 \%$ da amostra recebia até três salários mínimos como renda individual. Todos os participantes atingiram pontos de corte sugeridos por Brucki, Nitrini, Caramelli, Bertolucci e Okamoto (2003) no Mini Exame do Estado Mental - MEEM (Folstein, Folstein, \& McHugh, 1975; traduzido para uso no Brasil por Bertolucci, Brucki, Campacci, \& Juliano, 1994), que indicaram que os participantes não apresentavam perdas cognitivas indicativas de demência, e que, portanto, contavam com capacidade para responder aos instrumentos.

\section{Instrumentos}

Dados sociodemográficos. Questionário sociodemográfico para obter informações de descrição da 
composição da amostra, incluindo idade, escolaridade e estado civil.

Versão brasileira adaptada do California Older Person's Pleasant Events Schedule - OPPES-BR (Ferreira \& Barham, 2013). Esse instrumento foi produzido após estudos para avaliar a equivalência semântica, conceitual, cultural, idiomática e operacional entre a versão original e a versão brasileira do COPPES. A versão brasileira do instrumento é composta por 67 itens que descrevem atividades que idosos tendem a achar agradáveis. O respondente deve indicar a frequência com a qual realizou tais atividades no último mês, de acordo com a seguinte escala de pontuação: 0 (nunca); 1 (1 - 6 vezes), e 2 (mais de 7 vezes). O respondente também deve classificar o prazer subjetivo que experimentou ao realizar cada atividade, ou que experimentaria, caso a tivesse realizado, usando a seguinte escala de pontuação: 0 (não foi ou não teria sido agradável), 1 (foi ou teria sido razoavelmente agradá$v e l)$ e 2 (foi ou teria sido bastante agradável). O instrumento apresentou excelente consistência interna $(\alpha=0,95)$ e correlações negativas moderadas significativas com depressão, indicando evidências iniciais de validade interna e externa (Ferreira et al., 2015)

\section{Procedimento}

Os participantes foram contatados em locais frequentados por idosos (centros comunitários, Unidades Saúde Família, associações de aposentados) ou diretamente em suas residências. $\mathrm{O}$ convite e a explicação dos objetivos e condições da pesquisa foram feitos pessoalmente pela pesquisadora e por outra profissional treinada para contatar os participantes em potencial e aplicar os instrumentos. Caso o participante aceitasse colaborar, uma entrevista era previamente agendada e realizada pela pesquisadora ou pela profissional treinada. $\mathrm{Na}$ entrevista, o participante assinava o Termo de Consentimento Livre e Esclarecido. Depois dessa etapa, o idoso respondia primeiramente ao MEEM. Se a pontuação do instrumento indicasse ausência de comprometimento cognitivo indicativo de demência, o participante respondia ao OPPES-BR e ao questionário sociodemográfico. No caso do idoso não atingir a pontuação mínima no MEEM, o entrevistador agradecia sua participação, conversava sobre a importância de se envolver em eventos agradáveis, comunicava o resultado da aplicação do MEEM e recomendava a realização de uma avaliação neuropsicológica no caso do idoso ou algum membro familiar notarem outros sintomas indicativos de perdas cognitivas. As entrevistas ocorriam no local onde o idoso foi contatado ou em sua residência e duravam em média uma hora.

\section{Resultados}

\section{Análises Fatoriais Exploratórias}

Quando os autores do COPPES estabeleceram a estrutura fatorial dele, optaram por derivá-la dos escores totais obtidos pelos participantes, mas apenas em relação às pontuações de Agradabilidade (Rider et al., 2004). Os autores julgaram que derivar a estrutura fatorial a partir dos escores de Frequência poderia gerar algum viés, uma vez que, além da influência de interesse, a frequência de prática de atividades também é determinada pela disponibilidade de oportunidades para se engajar nessas atividades (Rider et al., 2004). Logo, as AFEs do instrumento original (COPPES-BR) foram efetuadas considerando-se apenas os escores totais de Agradabilidade.

Em seguida, para estabelecer também uma estrutura fatorial para os escores de Frequência, Rider et al. (2004) optaram por agregar os escores de frequência para os itens que compunham os domínios identificados anteriormente em relação a Agradabilidade e, então, calcular a consistência interna desses domínios, agora usando os escores de Frequência. Os autores constataram que os valores de consistência interna para cada domínio, considerando os escores de Frequência, eram adequados, concluindo que os domínios de atividades identificados anteriormente a partir da Agradabilidade eram também coerentes para a Frequência, além de serem apropriados para uso clínico, servindo como uma primeira categorização de eventos prazerosos (Rider et al., 2004). Essa parece ter sido a solução encontrada pelos autores originais para estabelecer apenas uma estrutura fatorial para um instrumento que apresenta, na verdade, duas subescalas (Frequência e Agradabilidade).

Levando-se em conta que Rider et al. (2004), utilizaram apenas uma subescala para derivar a estrutura fatorial do instrumento original e, considerando que as subescalas Frequência e Agradabilidade da versão brasileira do instrumento estavam altamente correlacionadas (Ferreira et al., 2015), optou-se por adotar a mesma abordagem de Rider et al. (2004) e, dessa forma, eleger apenas uma subescala para derivar a estrutura fatorial do instrumento. Isso a partir de uma subescala traz também outra vantagem prática, pois facilitaria o trabalho futuro do pesquisador ou do clínico que usar o OPPES-BR, uma vez que o conjunto de domínios de atividades a ser corrigido e interpretado será apenas um para o instrumento todo. No entanto, os pesquisadores do presente estudo julgaram ser mais adequado, primeiramente, investigar a estrutura fatorial de cada subescala, para então eleger uma que apresentasse a estrutura fatorial mais fácil de ser interpretada em termos teóricos e que apresentasse melhores propriedades psicométricas. Julgou-se igualmente prudente incluir, nessa análise, preliminar também a subescala derivada do produto entre Frequência e Prazer (Prazer Obtido), uma vez que esta terceira subescala era utilizada nos instrumentos que precederam o COPPES (MacPhillamy \& Lewinsohn, 1976; Teri \& Lewinsohn, 1982), além de ter sido anteriormente analisada nas investigações iniciais para buscar evidências de validade do OPPES-BR (Ferreira et al., 2015). 
Antes de dar início à AFE, foi verificado o valor da curtose e da assimetria dos escores de Frequência, Agradabilidade e Prazer Obtido, para cada item do instrumento, uma vez que a normalidade multivariada é um dos pressupostos para a AFE. De acordo com o critério adotado por Kline (2005), valores absolutos de assimetria $($ skew) devem ser inferiores a três $(|\mathrm{sk}|<3)$, e valores absolutos de curtose (kurtosis) devem ser inferiores a oito $(|\mathrm{ku}|<8)$. Corroborando os resultados anteriores da AFC (Ferreira et al., 2015), apenas o item 43 apresentou valores de curtose e assimetria elevados na subescala Agradabilidade. Esse item foi então excluído para iniciar o processo de AFE de todas as subescalas.

A AFE foi realizada utilizando-se o Programa IBM SPSS Statistics 20.0. O método de extração escolhido foi o da Fatorização do Eixo Principal, uma vez que esse método é usado para identificar fatores ou dimensões subjacentes que refletem o que as variáveis compartilham em comum, por assumir que cada variável é constituída por uma parte comum à estrutura fatorial e por uma parte específica da variável (Maroco, 2011).

Já havia sido constatado anteriormente por Ferreira et al. (2015), por meio da realização de uma análise fatorial confirmatória, que uma estrutura de cinco fatores parecia não ser a mais adequada para explicar a variação dos dados da amostra brasileira, tendo em vista que os apresentaram correlações altas entre si, indicando que a estrutura fatorial para a amostra brasileira provavelmente seria composta por menos de cinco fatores (Ferreira et al., 2015). Para testar esta hipótese, optou-se por iniciar a AFE, verificando primeiramente a adequação de uma solução de cinco fatores para as três subescalas gerais.

A adequabilidade da amostra à AFE (Fatorização do Eixo Principal) foi verificada por meio do índice de Kaiser-Meyer-Olkin (KMO) para as três subescalas (Frequência, Prazer e Prazer Obtido). Os valores indicaram que a análise fatorial era fortemente recomendada em todas as subescalas, tendo sido observado um valor de 0,928 para Agradabilidade; 0,926 para Frequência e 0,954 para Prazer Obtido. O método de rotação utilizado foi o Varimax para todas as subescalas. A solução de cinco fatores explicou $42 \%$ da variância total na subescala de Agradabilidade, 43\% na subescala de Frequência e 48\% na subescala de Prazer Obtido. No entanto, foram identificados muitos itens com saturações altas em mais de um fator, nas três análises, corroborando a hipótese levantada anteriormente de que fatores altamente correlacionados sinalizam a presença de muitos itens com cross-loadings, sugerindo uma estrutura fatorial composta por menos fatores (Ferreira et al., 2015).

Logo, optou-se então por testar a adequação de uma solução de quatro fatores. Essa solução explicou $40 \%$ da variância total na subescala de Agradabilidade, $40 \%$ na subescala de Frequência e 45\% na subescala de Prazer Obtido. As soluções fatoriais ainda apresentavam itens com cross-loadings, porém em quantidade menor do que a estrutura fatorial de cinco fatores. Nessa análise, preliminar nenhum item foi excluído da estrutura fatorial (com exceção do item 43 que apresentou valores de curtose e assimetria muito elevados e foi retirado logo no início da análise), pois o objetivo foi observar o comportamento de todos os itens numa estrutura fatorial de quatro fatores para todas as subescalas, e então eleger a subescala que apresentasse o menor número de anomalias, para então seguir com a AFE na subescala eleita. Optou-se também por manter o instrumento com uma estrutura de quateo fatores, pois, em termos práticos, seria mais útil que o instrumento discriminasse o maior número possível de categorias de atividades prazerosas, tanto para a prática clínica (gerar mais condições para o cliente ser capaz de variar os tipos de atividades em que se envolve) quanto para a prática de pesquisa (identificar variados tipos de atividades e com quais variáveis de interesse cada tipo pode estar associado).

Foram então analisadas as soluções fatoriais obtidas em cada subescala, tendo sido constatado que a solução mais facilmente interpretável do ponto de vista teórico e que apresentou menor quantidade de itens com cross-loadings foi a solução fatorial da subescala de Frequência. Considerando também que Ferreira et al. (2015) observaram que a subescala de Frequência apresentou correlações com variáveis de interesse, tais como grau de depressão, eleger a estrutura fatorial a partir dos escores de frequência parece ser a opção mais adequada para o contexto brasileiro.

Algumas outras razões também podem ser consideradas para justificar derivar a estrutura fatorial do instrumento adaptado a partir da subescala Frequência apenas. Primeiro, o COPPES é um instrumento composto por atividades potencialmente agradáveis, sendo mais provável que os respondentes assinalem as atividades como sendo agradáveis ao invés de desagradáveis. Por essa razão, a subescala Frequência discriminaria, melhor do que a subescala Agradabilidade, o envolvimento de idosos em atividades prazerosas. Segundo, para fins de intervenção, a subescala Frequência forneceria dados mais objetivos para guiar a prática terapêutica. Identificar os domínios em que o idoso apresenta maior e menor envolvimento em atividades seria mais útil para auxiliar o profissional a traçar um plano de intervenção.

Pelas razões elencadas anteriormente, optou-se por derivar a estrutura fatorial do OPPES-BR a partir dos escores da subescala Frequência. A AFE da subescala Frequência que testa a adequação de uma estrutura fatorial de quatro fatores será descrita em detalhes a seguir.

\section{AFE - Subescala Frequência}

Ao analisar a solução de quatro fatores para a subescala Frequência, primeiramente foi constatado que três itens apresentaram cargas fatoriais muito baixas. Foi adotado o critério de reter itens com cargas fatoriais acima de 0,3 (Hair, Anderson, Tatham, \& Black, 2005), portanto 
os itens 5, 32 e 47 foram descartados por não se adequarem a esse critério. Novamente foi rodada a AFE e, dessa vez, observou-se que os itens $12,6,28,48,51,21,29,14$, $67,61,3,4,24,15,58,64$ apresentaram cargas fatoriais altas em mais de um fator. De acordo com Reichenheim et al. (2014), umas das formas de se lidar com esse tipo de anomalia seria eliminando os itens que apresentam cross-loadings, desde que sejam ainda mantidos na estrutura fatorial, outros itens considerados adequados e capazes de mapear a dimensão do fator. Como se trata de uma escala extensa que abarca 67 itens e considerando que a AFC realizada anteriormente (Ferreira et al., 2015), apontou a existência de muitas correlações entre resíduos de itens de um mesmo fator (o que normalmente indica redundância entre os itens), considerou-se não ser problemático adotar o critério de eliminar todos os itens com cross-loadings nessa etapa da análise.

Logo, os itens 12, 6, 28, 48, 51, 21, 29, 14, 67, 61, 3, $4,24,15,58,64$ foram descartados e foi novamente rodada a AFE. Dessa vez, foi identificado que apenas os itens 7, 23, 30 e 44 apresentaram cross-loadings, porém com diferença maior que 0,1 entre as cargas fatoriais. Julgouse adequado manter esses itens dessa vez, considerando que a diferença entre as cargas fatoriais era aceitável e que os itens se adequavam ao fator do ponto de vista teórico (descreviam atividades que apresentavam funções bastante semelhantes às outras atividades do seu respectivo fator). Foram calculados, então, o valor da consistência interna para cada fator, sendo o alfa de Cronbach do Fator 1 igual a 0,94; para o Fator 2 igual a 0,83; para o
Fator 3 igual a 0,72 e para o Fator 4 igual a 0,7. Observouse que os valores de consistência interna variaram de adequados a excelentes (Hair et al., 2005). Essa nova solução apresentou índice de Kaiser-Meyer-Olkin (KMO) igual a 0,917 , e a variância total explicada foi de $43,6 \%$. Tal estrutura fatorial pode ser visualizada na Tabela 2 .

O Fator 1 foi composto por 18 itens e agregou eventos caracterizados por situações sociais em que o idoso exprime um senso de competência e utilidade para si e para os outros, além de autonomia e autoconfiança. Os 14 itens do Fator 2 descreveram atividades mais contemplativas e de introspecção, que poderiam envolver contato com a natureza e expressão de sentimentos positivos. Já o Fator 3 foi composto por nove itens e agregou atividades práticas relacionadas ao envolvimento com a comunidade, às atividades domésticas e também da própria rotina do idoso. Por fim, o Fator 4 agregou três itens que descreveram atividades intelectuais, em que são necessárias habilidades de leitura e escrita para se envolver.

Como se pode observar na Tabela 2, todos os itens apresentaram saturação igual ou superior a 0.3 , critério mínimo para reter um item no fator (Hair et al., 2005). Os valores das comunalidades revelaram-se aceitáveis, embora os itens 17, 18 e 56 demonstrassem valores abaixo de 0,3 . Itens com comunalidades mais baixas foram mantidos, uma vez que os valores de alfa de Cronbach dos fatores eram adequados. A estrutura fatorial final da subescala Frequência do OPPES-BR foi composta por 44 itens.

Tabela 2

Análise Fatorial no Método da Fatorização pelo Eixo Principal da Subescala Frequência do OPPES-BR

\begin{tabular}{|c|c|c|c|c|c|}
\hline Itens & Fator1 & Fator2 & Fator3 & Fator4 & $h^{2}$ \\
\hline 39. Fazer uma nova amizade & 0,79 & & & & 0,70 \\
\hline 53. Conhecer novos lugares & 0,73 & & & & 0,65 \\
\hline 54. Aprender sobre novos assuntos & 0,71 & & & & 0,61 \\
\hline 52. Conhecer novas pessoas do mesmo sexo & 0,69 & & & & 0,68 \\
\hline 40. Ser requisitado a ajudar ou a oferecer um conselho & 0,68 & & & & 0,68 \\
\hline 36. Ter uma ideia criativa & 0,67 & & & & 0,60 \\
\hline 63. Comprar uma roupa nova & 0,62 & & & & 0,54 \\
\hline 19. Ver coisas boas acontecerem com familiares ou amigos & 0,62 & & & & 0,56 \\
\hline 62. Ouvir música clássica & 0,62 & & & & 0,48 \\
\hline 26. Ouvir alguém dizer que sou importante & 0,61 & & & & 0,56 \\
\hline 23. Dizer algo de forma clara & 0,60 & 0,30 & & & 0,63 \\
\hline 16. Ser elogiado por pessoas que admiro & 0,60 & & & & 0,63 \\
\hline 07. Ter uma conversa franca e aberta & 0,58 & 0,36 & & & 0,55 \\
\hline 45. Aconselhar outras pessoas com base em experiências passadas & 0,57 & & & & 0,60 \\
\hline 31. Terminar uma tarefa difícil & 0,57 & & & & 0,48 \\
\hline $\begin{array}{l}\text { 49. Sair da cidade } \\
\text { (ir para chácara sítio fazenda praia etc) }\end{array}$ & 0,56 & & & & 0,48 \\
\hline
\end{tabular}


Tabela 2 (continuação)

Análise Fatorial no Método da Fatorização pelo Eixo Principal da Subescala Frequência do OPPES-BR

\begin{tabular}{|c|c|c|c|c|c|}
\hline Itens & Fator1 & Fator2 & Fator3 & Fator4 & $h^{2}$ \\
\hline 59. Visitar um museu, uma biblioteca ou outra atração cultural & 0,50 & & & & 0,46 \\
\hline 10. Tomar café, chá, etc., com amigos & 0,49 & & & & 0,37 \\
\hline $\begin{array}{l}\text { 65. Planejar ou organizar alguma coisa } \\
\text { (Ex.: uma reunião familiar, uma festa, um evento, etc.) }\end{array}$ & 0,46 & & & & 0,46 \\
\hline 41. Procurar produtos em promoção & 0,43 & & & & 0,45 \\
\hline 02. Estar com amigos & 0,33 & & & & 0,33 \\
\hline 30. Pensar em pessoas das quais eu gosto & & 0,61 & 0,35 & & 0,54 \\
\hline $\begin{array}{l}\text { 09. Ouvir sons da natureza } \\
\text { (Ex.: canto dos pássaros, vento, chuva, etc.) }\end{array}$ & & 0,57 & & & 0,51 \\
\hline 66. Sorrir para as pessoas & & 0,57 & & & 0,48 \\
\hline 38. Ouvir pássaros cantando & & 0,53 & & & 0,49 \\
\hline 25. Olhar para as estrelas ou para a lua & & 0,52 & & & 0,49 \\
\hline 37. Ter momentos de sossego & & 0,52 & & & 0,57 \\
\hline 11. Pensar em mim & & 0,52 & & & 0,39 \\
\hline 22. Olhar ou cheirar uma flor ou uma planta & & 0,48 & & & 0,43 \\
\hline 44. Demonstrar o meu afeto a alguém & 0,32 & 0,47 & & & 0,50 \\
\hline 01. Olhar para o céu & & 0,47 & & & 0,42 \\
\hline 50. Ter um tempo livre & & 0,44 & & & 0,54 \\
\hline 35. Estar com alguém que amo & & 0,37 & & & 0,30 \\
\hline 55. Manter a casa limpa & & 0,33 & & & 0,33 \\
\hline 18. Ouvir música & & 0,30 & & & 0,24 \\
\hline 13. Fazer trabalho voluntário & & & 0,54 & & 0,51 \\
\hline 27. Trabalhar em um projeto comunitário & & & 0,50 & & 0,51 \\
\hline 57. Ir à igreja, ao templo, etc. & & & 0,43 & & 0,37 \\
\hline $\begin{array}{l}\text { 08. Fazer bem uma tarefa } \\
\text { (Ex.: cozinhar bem algum prato, fazer bem algum conserto doméstico, etc.) }\end{array}$ & & & 0,42 & & 0,47 \\
\hline 33. Cozinhar porque me sinto inspirado & & & 0,42 & & 0,51 \\
\hline 20. Colecionar receitas & & & 0,38 & & 0,39 \\
\hline 60. Planejar minha rotina diária & & & 0,35 & & 0,33 \\
\hline 17. Rezar, orar ou meditar & & & 0,34 & & 0,29 \\
\hline 56. Fazer artesanato & & & 0,33 & & 0,28 \\
\hline 42. Ler revistas & & & & 0,69 & 0,52 \\
\hline 34. Fazer leituras & & & & 0,61 & 0,44 \\
\hline 46. Solucionar um problema, palavra-cruzada, jogo de raciocínio & & & & 0,49 & 0,32 \\
\hline \% de Variância Total Explicada & 28 & 6,5 & 5 & 4,1 & \\
\hline Valores próprios & 13,2 & 3 & 2,3 & 2 & \\
\hline Alfa de Cronbach & 0,94 & 0,83 & 0,72 & 0,7 & \\
\hline
\end{tabular}

$\mathrm{Na}$ Tabela 3, podem ser visualizadas as correlações entre os quatro fatores da subescala Frequência. Observa-se que os valores indicaram correlações fracas e moderadas estatisticamente significativas, não existindo correlações altas entre os fatores.

Adotando a mesma abordagem dos autores originais do COPPES (Rider et al., 2004), foram também calculados os valores de consistência interna para a subescala Agradabilidade, considerando os domínios derivados a partir da subescala Frequência. Foi constatado que os valores de consistência interna também variaram de adequados a excelentes para a subescala Agradabilidade: Fator 1=0,90; Fator 2=0,86; Fator $3=0,75$; Fator $4=0,72$. 
Tabela 3

Matriz de Correlações dos Fatores da Subescala Frequência

\begin{tabular}{|c|c|c|c|}
\hline & 2. & 3. & 4. \\
\hline 1. Atividades Sociais e de Competência & $0,59^{* *}$ & $0,53^{* *}$ & $0,29^{* *}$ \\
\hline 2. Atividades Contemplativas & -- & $0,45^{* *}$ & $0,24^{* *}$ \\
\hline 3. Atividades Práticas & -- & -- & $0,27^{* *}$ \\
\hline 4. Atividades Intelectuais & -- & -- & -- \\
\hline
\end{tabular}

\section{Discussão}

No presente estudo, foi descrito o processo de validação preliminar do OPPES-BR que avalia Frequência e Agradabilidade do envolvimento de idosos em atividades. Considerando que resultados anteriores da AFC demonstraram que a subescala Frequência e Agradabilidade quando avaliadas por uma amostra brasileira não abarcavam domínios idênticos aos da amostra americana (Ferreira et al., 2015), procedeu-se, dessa forma, uma AFE cujos resultados sugeriram que a versão brasileira das subescala Frequência se divide em quatro fatores: F1 - Atividades Sociais e de Competência; F2 Atividades Contemplativas; F3 - Atividades Práticas; e F4 - Atividades Intelectuais. Julgou-se ser mais adequado derivar a estrutura fatorial do OPPES-BR a partir dos escores na subescala Frequência, uma vez que a solução fatorial dela era mais facilmente interpretável do ponto de vista teórico, demonstrou melhores propriedades psicométricas, além de fornecer dados mais relevantes para uso em contexto clínico.

O Fator 1 (Atividades Sociais e de Competência) inclui atividades que descreveram interações do idoso com seu ambiente e também com outras pessoas. Tais atividades parecem ter a função de socializar, mas também exprimem um senso de competência, utilidade, autoconfiança e autonomia do idoso no que concerne ao relacionamento com seu meio (viajar, conhecer novos lugares, visitar museu, aprender novos assuntos, etc.) e também com as outras pessoas (ser elogiado, fazer uma nova amizade, dizer algo de forma clara, ser requisitado a ajudar e dar conselhos, etc.). Esse fator foi nomeado como Atividades Sociais e de Competência, por descrever atividades que exigem iniciativa, autonomia e certo grau de competência para que o idoso interaja com seu ambiente e com outras pessoas. Esses resultados corroboram um estudo que investigou as percepções de idosos brasileiros sobre o envelhecimento, onde foi identificado que o idoso relaciona suas atividades sociais (interações com familiares e amigos) com um senso de competência e utilidade, percebendo-se bastante capaz de contribuir com outras pessoas e com a sociedade, por meio das relações sociais que mantém (Moimaz, Almeida, Lolli, Garbin, \& Saliba, 2009).

Interessante notar que alguns itens que fazem parte do Fator 1 saturaram nos domínios socializing, being effective e relaxing da versão americana. Atividades que apresentaram função diferenciada no contexto americano parecem ter apresentado uma única função no contexto brasileiro, por terem saturado em um mesmo domínio. Ou seja, pode ser que na cultura brasileira, atividades de socializar, relaxar, sentir-se útil e competente com relação a si mesmo e com os outros parecem fazer parte de uma classe maior, designado aqui como atividades sociais e de competência.

O Fator 2 (Atividades Contemplativas) descreveram atividades de contato com a natureza e também de expressão de sentimentos positivos para com outras pessoas, porém, em uma perspectiva mais contemplativa e introvertida. Esse fator incluiu atividades que pertenciam ao domínio relaxing do instrumento americano, porém apenas as atividades de contato com a natureza e que não pressupõem envolvimento com outras pessoas. Também incluiu atividades da subescala contemplating e algumas da socializing. No entanto, analisando o conjunto total dos itens que saturaram no Fator 2, parece ser mais adequado supor que, no contexto brasileiro, tais atividades teriam mais uma função de introversão, contemplação e recolhimento, porém em que seria também possível expressar sentimentos positivos (por exemplo: pensar nas pessoas que gosto), mas que não necessariamente especificam comportamentos de socialização. A determinação do fator Atividades Contemplativas como um tipo de atividade prazerosa ao idoso brasileiro é corroborada pelo estudo de Carmona, Couto, e Scorsolini-Comin (2014), que constataram, a partir do relato de uma amostra de idosas mineiras, que a prática de atividades que não envolvem a presença de outras pessoas pode também ser muito prazerosa, pois é um momento em que o indivíduo tem a oportunidade de olhar para si, reviver boas memórias e obter satisfação num momento que é apenas seu.

O Fator 3 (Atividades Práticas) descreveu atividades de envolvimento com a comunidade (ex.: fazer trabalho voluntário ou comunitário, ir à igreja), mas também outras atividades que podem fazer parte da rotina doméstica (cozinhar, colecionar receitas, planejar a rotina). A maioria dos itens que saturaram no Fator 3 faz parte também da subescala doing do instrumento americano, que descreve eventos em que o idoso parece manter-se ativo e envolvido em atividades práticas relevantes para si e que também repercutem em sua comunidade. Esse fator foi o que mais se assemelhou a algum domínio previamente 
identificado na escala americana, sugerindo que atividades desse tipo podem apresentar funções semelhantes tanto no contexto brasileiro como no americano.

Por fim, o Fator 4 (Atividades Intelectuais) foi composto por apenas quatro atividades, porém é o fator que pareceu mais se diferenciar dos outros fatores pelo fato de ter apresentado uma fraca correlação com os demais. O que pareceu diferir o Fator 4 dos restantes é que este incluiu apenas atividades em que o idoso precisa saber ler e escrever para estar envolvido. Interessante notar que esse domínio de atividades foi identificado na amostra brasileira, mas não na amostra americana. Esse fato se deve provavelmente à diferença entre Brasil e EUA com relação à taxa de idosos analfabetos que é bem maior no Brasil (Rebouças \& Pereira, 2008). Logo, saber ler e escrever não seriam habilidades determinantes a ponto de diferenciar um domínio de atividades no contexto americano, como foi no Brasil.

Uma pesquisa brasileira (Massi et al., 2015) corrobora o achado do presente estudo de que atividades que envolvam leitura e escrita podem ser vivenciadas pelo idoso como um tipo específico de atividade associado a sentimentos positivos. Massi et al. (2015) analisaram os efeitos que atividades de leitura e escrita podem gerar no bem-estar e autonomia em uma amostra de oito idosos, e identificaram no discurso desses participantes que esse tipo de atividade se associa a um reconhecimento e valorização na esfera pessoal e social, além de sentimentos de autonomia e utilidade.

Consideramos não ter sido problemática a eliminação de itens que apresentaram valores de curtose e assimetria elevados (43 - sentir uma presença divina), saturações baixas nos fatores ( 5 - fazer compras; 32- divertir pessoas; 47- fazer arranjos de flores) e cargas elevadas em mais de um fator (3 - pessoas demonstrarem interesse no que eu tenho a dizer; 4 - pensar em recordações agradáveis; 6 - apreciar paisagens bonitas; 12 - alguém me elogiar ou dizer que fiz bem alguma coisa; 14 - planejar férias ou viagens; 15 - abraçar, beijar, demonstrar afeto; 21 - realizar um plano ou projeto do meu jeito; 28 - elogiar ou parabenizar alguém; 29- assistir ao pôr-do-sol; 48 - oferecer ajuda a alguém; 51 - sentir que alguém precisa de mim; 58 - sentir-se amado; 61 - estar com pessoas alegres; 64 - fazer um balanço da minha vida; 67 - estar perto da natureza). Embora tenham sido eliminados 23 itens no total, a AFC realizada anteriormente demonstrou a presença de muitos itens redundantes (Ferreira et al., 2015), sugerindo que, mesmo com a eliminação de itens problemáticos, os itens restantes ainda seriam capazes de mapear a dimensão de cada fator sem perder informação. Por esse motivo, resolveu-se ser rigoroso na remoção de itens que apresentavam essa anomalia e, dessa forma, garantir uma estrutura fatorial mais robusta para o instrumento.

Outra importante justificativa para se ter uma escala reduzida, refere-se ao seu uso para pesquisa. Para fins de investigação, seria mais prático e adequado contar com uma versão reduzida da escala, mas que fosse ainda capaz de captar os domínios relevantes de atividades prazerosas. Já para fins de intervenção, seria adequado utilizar a versão completa da escala, uma vez que o instrumento é aplicado também com a função de ajudar o idoso a discriminar atividades em que goste de se envolver. Portanto, quanto mais dicas ele obtiver sobre eventos potencialmente agradáveis, maiores as chances de ele conseguir também pensar em outros eventos agradáveis que possa praticar e que não se restrinjam ao instrumento. Porém, para calcular a pontuação nos fatores, deveriam ser considerados apenas os itens da versão reduzida produzida após as AFEs.

$O$ fato de ter sido constatado que as versões brasileira e americana do COPPES não apresentam a mesma estrutura fatorial (Ferreira et al., 2015) levou a adotar uma abordagem universalista no que concerne ao processo de adaptação transcultural do COPPES para o Brasil (Herdman, Fox-Rushby, \& Badia, 1997). Isso significa que envolvimento de idosos em atividades prazerosas é um construto influenciado por fatores culturais e, portanto, é esperado que esse construto se manifeste de maneira distinta em um e em outra cultura. Talvez a diferença cultural entre Brasil e EUA que tenha ficado mais evidente nos resultados do presente estudo seja o fato do OPPES-BR incluir um domínio que contemple apenas atividades de leitura e escrita, ao passo que esse domínio não aparece na estrutura fatorial americana pelo fato da taxa de idosos analfabetos nos EUA ser menor.

No entanto, mesmo adotando uma abordagem universalista na adaptação do COPPES para o Brasil, ainda seria possível a realização de estudos transculturais utilizando as versões do COPPES. Nesse caso, seria possível comparar apenas os escores globais das subescalas mantendo todos os itens iniciais (Frequência, Agradabilidade e Prazer Obtido). Porém, não seria possível estabelecer comparações no que concerne à estrutura fatorial do instrumento. Talvez o mais relevante fosse analisar as diferenças e similaridades sobre como tal construto se manifesta em cada cultura, e acredita-se que os resultados do presente estudo fornecem vislumbres iniciais sobre essa questão.

Os resultados deste estudo devem ser lidos levando-se em conta algumas limitações metodológicas. Primeiramente, a amostra do estudo não pode ser considerada uma amostra representativa de idosos brasileiros, sendo possível apenas considera-la representativa do município onde foram coletados os dados. Além disso, a estrutura fatorial brasileira necessita ainda de ser confirmada. Logo, é necessária a realização de análises fatoriais confirmatórias em investigações futuras, utilizando-se de outra amostra independente de idosos com características semelhantes da deste estudo, seguindo assim a recomendação de Reichenheim et al. (2014) no que concerne aos passos para adaptar um instrumento para outra cultura. Entretanto, já se pode considerar que os dados obtidos constituem algumas evidências de validade 
da estrutura interna do instrumento. Espera-se que, no futuro, o OPPES-BR possa ser utilizado em pesquisas e intervenções que visam a promover o bem-estar de idosos no Brasil.

\section{Referências}

Alvarenga, M. R. M., Oliveira, M. A. C., \& Faccenda, O. (2012). Sintomas depressivos em idosos: análise dos itens da Escala de Depressão Geriátrica. Acta Paulista de Enfermagem, 25(4), 497-503. doi: 10.1590/S0103-21002012000400003

Bertolucci, P. H. F., Brucki, S. M. D., Campacci, S. R., \& Juliano, Y. (1994). The mini-mental state examination in a general population: Impact of educational status. Arquivos de Neuropsiquiatria, 52(1), 1-7. Recuperado de http://www.ncbi.nlm.nih.gov/pubmed/8002795

Bouman, T. K., \& Luteijn, F. (1986). Relations between the pleasant events schedule, depression and other aspects of psychopathology. Journal of Abnormal Psychology, 95(4), 373-377. doi: 10.1037/0021-843X.95.4.373

Brucki, S. M. D., Nitrini, R., Caramelli, P., Bertolucci, P. H. F., \& Okamoto, I. H. (2003). Suggestions for utilization of the mini-mental state examination in Brazil. Arquivos de Neuropsiquiatria, 61(3), 777-781. doi: 10.1590/S0004-282X2003000500014

Carmona, C. F., Couto, V. V. D., \& Scorsolini-Comin, F. (2014). A experiência de solidão e a rede de apoio social de idosas. Psicologia em Estudo, 19(4), 681-691. doi: 10.1590/1413-73722395510

Ferreira, H. G., \& Barham, E. J. (2011). O envolvimento de idosos em atividades prazerosas: revisão de literatura sobre instrumentos de aferição. Revista Brasileira de Geriatria e Gerontologia, 14(3), 579-590. doi: 10.1590/S1809-98232011000300017

Ferreira, H. G., \& Barham, E. J. (2013). Adaptação transcultural de um instrumento para avaliar o envolvimento de idosos em atividades prazerosas. Cadernos de Saúde Pública, 29(12), 2554-2560. doi: 10.1590/0102-311X00130212

Ferreira, H. G., Barham, E. J., \& Fontaine, A. M. G. V. (2015). A measure to assess Brazilian older people's involvement in pleasant activities: Initial evidence of internal and external validity. Clinical Gerontologist, 38(5), 375-394. doi: 10.1080/07317115.2015.1067270

Folstein, M. F., Folstein S. E., \& McHugh P. R. (1975). Mini-Mental state: A practical method for grading the cognitive state of patients, for clinicians. Journal of Psychiatric Research, 12(3), 189-198. Recuperado de http://www.ncbi.nlm.nih.gov/pubmed/1202204

Fundação SEADE (2010). Índice paulista de vulnerabilidade social. Recuperado de http://www.seade.gov.br/analises_estudos/indice-paulista-devulnerabilidade-social-ipvs-versao-2010/

Hair, J. F., Black, W., Anderson, R. E., \& Tatham, R. L. (2005). Multivariate Data Analysis (5th ed). New Jersey: Prentice-Hall.

Herdman, M., Fox-Rushby, J., \& Badia, X. (1997). "Equivalence" and the translation and adaptation of health-related quality of life questionnaires. Quality of Life Research, 6(3), 237-247. doi: 10.1023/A:1026410721664

Kline, R. B. (2005). Principles and practice of structural equation modeling (2nd ed.). New York: Guilford.

Lewinsohn, P. M., \& Graf, M. (1973). Pleasant activities and depression. Journal of Consulting and Clinical Psychology, 41(2), 261-268. Recuperado de http://www.ncbi.nlm.nih.gov/pubmed/4147832

Lima, M. T. R., Silva, R. S., \& Ramos, L. R. (2009). Fatores associados à sintomatologia depressiva numa coorte urbana de idosos. Jornal Brasileiro de Psiquiatria, 58(1), 1-7. doi: 10.1590/S0047-20852009000100001

Massi, G., Berberian, A. P., Guarinello, A. C., Lourenço, R. C., Tonocchi, R., \& Neto, J. S.(2015). Linguagem e envelhecimento: práticas de escrita autobiográfica junto a idosos. Revista CEFAC, 17(6), 2065-2071. doi: 10.1590/1982-021620151764615

Moimaz, S. A. S., Almeida, M. E. L., Lolli, L. F., C. A. S., Garbin, C. A. S., \& Saliba, N. A. (2009). Envelhecimento: análises de dimensões relacionadas à percepção dos idosos. Revista Brasileira de Geriatria e Gerontologia, 12(3), 361-375. doi: 10.1590/1809-9823.2009.00005

MacPhillamy, D. J., \& Lewinsohn, P. M. (1976). Manual for the pleasant events schedule [Mimeo]. Universidade de Oregon, Eugene, OR, EUA. Recuperado de http://www.ori.org/files/Static\%20Page\%20Files/PESManual.pdf

Maroco, J. (2010). Análise de equações estruturais (1ª ed.). Pero Pinheiro: Report Number.

Maroco, J. (2011). Análise Estatística com o SPSS Statistics (5 ed.). Pero Pinheiro: Report Number

Meeks, S., Young, C.M., \& Looney, S.W. (2007). Activity participation and affect among nursing home residents: Support for a behavioural model of depression. Aging \& Mental Health, 11(6), 751-760. doi: 10.1080/13607860701546910

Nogueira, E. L., Rubin, L. L., Giacobbo, S. S., Gomes, I., \& Neto, A. C. (2014). Rastreamento de sintomas depressivos em idosos na Estratégia Saúde da Família, Porto Alegre. Revista de Saúde Pública, 48(3), 368-377. doi: 10.1590/S0034-8910.2014048004660

Rebouças, M., \& Pereira, M.G. (2008). Health indicators in the elderly: A comparison of Brazil and the United States. Panamerican Journal of Public Health, 23(4), 237-246. doi: 10.1590/S1020-49892008000400003

Reichenheim, M. E., Hokerber, Y. H. M., \& Moraes, C. L. (2014). Assessing construct validity of epidemiological measurement tools: A seven-step roadmap. Cadernos de Saúde Pública, 30(5), 927-939. doi: 10.1590/0102-311X00143613

Rider, K., Gallagher-Thompson, D., \& Thompson, L. (2004). California Older Person's Pleasant Events Schedule: Manual [Manual]. Universidade de Stanford, Palo Alto, CA, EUA. Recuperado de http://med.stanford.edu/oafc/coppes_files/Manual2.pdf

Rider, K., Gallagher-Thompson, D., \& Thompson, L. (2016). California Older Person's Pleasant Events Schedule: A tool to help older adults increase positive experiences. Clinical Gerontologist, 39(1), 64-83. doi: 10.1080/07317115.2015.1101635

Teri, L., \& Lewinsohn, P. (1982). Modification of the pleasant and unpleasant events schedules for use with the elderly. Journal of Consulting and Clinical Psychology, 50(3), 444-445. doi: 10.1037/0022-006X.50.3.444

recebido em julho de 2016 aprovado em maio de 2017

\section{Sobre as autoras}

Heloísa Gonçalves Ferreira atualmente é professora adjunta do Departamento de Psicologia da Universidade Federal do Triângulo Mineiro. Doutora e Mestre em Psicologia pelo Programa de Pós-Graduação em Psicologia- área de concentração: comportamento e cognição - da Universidade Federal de São Carlos. Especialista em Psicoterapia Comportamental pelo Instituto de Terapia por Contingências de Reforçamento - ITCR de Campinas-SP.

Elizabeth Joan Barham graduada em Psicologia pela Bishop's University, mestrado em Psicologia Social na University of Waterloo e doutorado em Psicologia Social e de Desenvolvimento na University of Guelph. Atualmente é docente no Departamento de Psicologia na Universidade Federal de São Carlos. 